\title{
Computer simulation study of irreversible adsorption: Coverage fluctuations
}

\author{
Jordi Faraudo* and Javier Bafaluy ${ }^{\dagger}$ \\ Departament de Física, Grup de Fisica Estadistica, Facultat de Ciencies Edifici Cc, Universitat Autònoma de Barcelona, \\ E-08193 Bellaterra, Barcelona, Spain
}

(Received 04 August 2001; published 7 February 2002)

\begin{abstract}
In this paper, we develop a cellular automata model to study the coverage fluctuations in monolayers of irreversible adsorbed particles. The effect of bulk diffusion and excluded volume interactions between adsorbed and incoming particles on coverage fluctuations is analyzed by simulations and analytically. We also show that the macroscopic boundary and initial conditions imposed at the system (open or closed cell) determine the effect of these factors on coverage fluctuations. In fact, under certain conditions, the excluded volume interactions only influence fluctuations near the jamming limit.
\end{abstract}

DOI: 10.1103/PhysRevE.65.037101

PACS number(s): 05.10.-a, 82.70.Dd, 68.43.Mn, 05.40.-a

The irreversible adsorption of colloidal particles (macromolecules, latexes, bacteria, etc.) from fluid suspensions to solid surfaces is a complex phenomenon of great interest (for example, in filtration, chromatography,...). Much effort has been devoted to the study of the effect of transport mechanisms on the adsorption kinetics and on the structure of the adsorbed monolayer [1,2]. Recently, both experimental [3-5] and theoretical [6] studies have analyzed also the fluctuations in the number of adsorbed particles. It is expected that coverage fluctuations reveal valuable information about the adsorption process. However, the experimental results [3-5] are difficult to interpret without a theory that can take into account the effect of bulk diffusion. Up to now, theoretical results concerning fluctuations have been developed in the framework of geometrical models based on the surface excluded by adsorbed particles [6]. These models do not consider the transport of the particles form the bulk towards the surface. Thus, the influence of bulk diffusion on coverage fluctuations is not known.

In this article, we develop a cellular automata model (CA) in order to analyze coverage fluctuations in irreversible adsorption driven by diffusion. Two main reasons justify the convenience of CA models in diffusion problems [7]: (a) it is possible to develop computer simulations with a reasonable effort (they require less computational resources than other techniques) and (b) their analytical tractability. Our goal in this paper is to determine, within this CA model, the role on coverage fluctuations of (a) bulk diffusion and (b) the excluded volume interactions between incoming and adsorbed particles. Also, we show that the relative effect of each of these factors strongly depends on the boundary and initial conditions imposed on the system. This important effect was not predicted in previous studies and should be taken into account in order to interpret properly the experimental results.

The CA model consists of a square adsorbing surface (labeled as $j=0$ ) with $N_{\max }$ adsorbing sites and a bulk phase $\left(j=1, \ldots, L_{z}\right)$ with $V=N_{\max } L_{z}$ sites. Each site can allocate only one particle. At each time step, all diffusing particles randomly select with equal probability $p$ an adjacent node $(p=1 / 6)$. If the selected node is free, a move to this node is performed, but if it is occupied, the particle remains at its initial position. When a particle reaches a free site at the adsorbing surface, it is irreversibly adsorbed and remains immobilized at this site. The process ends when the jammed state is reached (all the sites at the adsorbing surface are occupied) or when all particles are adsorbed. We consider periodic boundary conditions on axis $x$ and $y$. On the $z$ axis we consider two kinds of conditions: (a) a reflecting barrier at $j=L_{z}$ (closed cell conditions), and (b) an equilibrium reservoir with a fixed number of particles $N_{R}$ maintained at $j$ $=L_{z}$ (open cell conditions). This reservoir is maintained by removing or adding particles if necessary at each time step. The initial condition $(t=0)$ is a uniform distribution of $N_{B}$ particles in the case of closed cell conditions and an empty system in the case of open cell conditions.

The number of adsorbed particles $N_{0}(t)$ increases monotonically with time due to the irreversible nature of the adsorption process. However, $N_{0}(t)$ presents statistical fluctuations: at a given time $t$, identical surfaces with the same boundary and initial conditions may have different number of adsorbed particles. Thus, we define $\bar{N}_{0}(t)$ as the mean number of adsorbed particles averaged over an ensemble of realizations of the adsorption process (with the same macroscopic boundary and initial conditions). The coverage $\theta$ is defined as the mean fraction of the surface covered by particles $\theta(t) \equiv \bar{N}_{0}(t) / N_{\max }$. It increases monotonically with time from $\theta(0)=0$ to its maximum value $\theta(t \rightarrow \infty)=1$ (saturation) due to irreversible adsorption. We also define $\bar{N}_{j}(t)$ as the mean number of diffusing particles at the plane $j$ at time $t$. The mean fraction of occupied sites at slab $j$ is $n_{j}(t)$ $=\bar{N}_{j}(t) / N_{\max }$. The mean flux of adsorbing particles towards the surface is defined as $J_{S}(t) \equiv \theta(t+1)-\theta(t)$. Typically, in adsorption studies, one characterizes the adsorbed particle number fluctuations by the reduced variance $V_{r}$ defined as

\footnotetext{
*Electronic address: jordi@circe.uab.es

${ }^{\dagger}$ Electronic address: Javier.Bafaluy@uab.es
}

$$
V_{r}(t) \equiv \frac{\bar{N}_{0}^{2}-\bar{N}_{0}^{2}}{\bar{N}_{0}}=\frac{\sigma^{2}}{\bar{N}_{0}}
$$


TABLE I. Sets of values for the simulation parameters.

\begin{tabular}{cccccccc}
\hline \hline & BC & $L_{z}$ & $N_{\max }$ & $N_{B}$ & $n_{j}(t=0)$ & $n_{R}$ & $\theta(t \rightarrow \infty)$ \\
\hline$S 1$ & Closed & 100 & $10^{6}$ & $2 \times 10^{6}$ & 0.02 & & 1 \\
$S 2$ & Closed & 50 & $10^{4}$ & 11000 & 0.022 & & 1 \\
$S 3$ & Closed & 5 & $10^{4}$ & 4020 & 0.0804 & & 0.402 \\
$S 4$ & Open & 4 & $10^{4}$ & 0 & 0 & 0.01 & 1 \\
$S 5$ & Open & 50 & $10^{4}$ & 0 & 0 & 0.022 & 1 \\
\hline \hline
\end{tabular}

Now, our objective is to investigate the effect on $V_{r}$ of diffusion and the interaction between incoming and adsorbed particles on the CA model.

We have performed computer simulations of the CA model both with open and closed cell conditions and we have monitored $\theta(t), J_{S}(\theta)$, and $V_{r}(\theta)$. The values of the simulation parameters employed are summarized in Table I. For each set of parameters (labeled as $S 1, S 2$, etc.), we have performed 10000 independent runs. All simulations correspond to suspensions that are diluted enough in order to make irrelevant the excluded volume effects at the bulk. $S 1$ to $S 3$ correspond to closed cell simulations with different number of initial particles: in $S 1$ we have $N_{B} \gg N_{\max }$, in $S 2$ we have $N_{B} \sim N_{\max }$, and in $S 3 N_{B} \ll N_{\max } . S 4$ and $S 5$ correspond to an open cell with the particle reservoir near and far from the adsorbing surface, respectively.

Adsorption kinetics. The flux of adsorbing particles obtained in simulations is shown in Fig. 1. In order to understand more deeply the results of simulations, we have developed a mean field (MF) description of the CA. In this description, the flux obeys the balance equation,

$$
J_{S} \equiv \theta(t+1)-\theta(t)=p \phi(\theta) n_{1}(t) .
$$

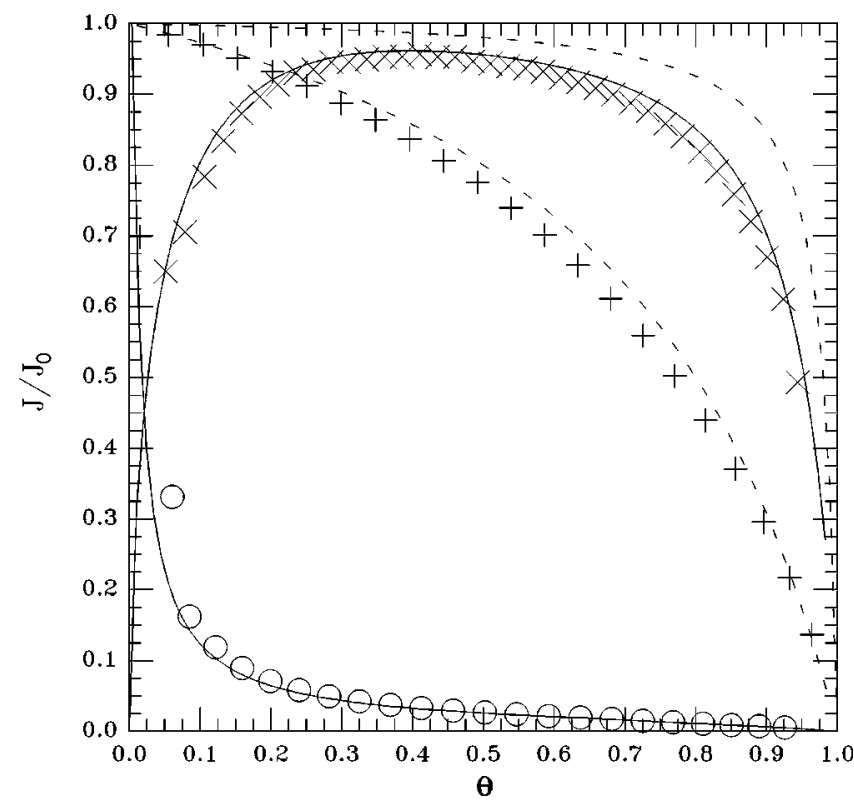

FIG. 1. Normalized flux of particles: simulations $S 2(\bigcirc), S 4$ $(+)$, and $S 5(\times)$, quasisteady analytical solution (dashed line), and non-steady numerical solution of MF equations (solid line).
Equation (2) expresses the fact that $\theta$ increases due to the particles at $j=1$ that move to $j=0$. The probability of this move is $p=1 / 6$ and the probability of finding a free site to adsorb in a move is (in a "mean field" sense) the fraction of free sites, $\phi=1-\theta$. At slab $j=1$, particles can arrive from $j=2$ and exit to $j=0,2$. Thus, a balance for the number of particles at this slab leads to

$$
n_{1}(t+1)-n_{1}(t)=p n_{2}(t)-p n_{1}(t)-p \phi(\theta) n_{1}(t) .
$$

In Eq. (3) it has been assumed that bulk concentration is small, so we do not take into account the fact that each site at $j=1$ can only be occupied by one particle. In a completely analogous way, we can obtain the balance equations for the mean number of particles at each slab $j$,

$$
\begin{aligned}
n_{j}(t+1)-n_{j}(t) & =-2 p n_{j}(t)+p n_{j+1}(t)+p n_{j-1}(t), \\
2 & \leqslant j<L_{z} .
\end{aligned}
$$

Closed cell conditions. In this case, the set of equations (2)-(4) has been solved numerically with the initial condition $n_{j}(0)=N_{B} / V(j \geqslant 1)$ and the perfectly reflecting boundary condition $n_{L_{z}}(t)=n_{L_{z-1}}(t)$. The calculated flux of particles is in very good agreement with the flux computed from simulations as shown in Fig. 1 for the case $S 2$. Initially, the flux is given by $J_{0}=p N_{B} / L_{z}$ and decreases with $\theta$, vanishing at the jamming limit $\theta=1$.

Open cell conditions. In this case, we consider the initial condition of an empty system, $n_{j}(0)=0\left(j<L_{z}\right)$ and the boundary condition of a particle reservoir: $n_{L_{z}}(t)=n_{R}$. The set of equations (2)-(4) with these conditions has been solved numerically. Also, we note that for times larger than the characteristic diffusion time $\tau \sim L_{z}^{2}$ these equations admit an analytical solution using the quasistationary approximation $n_{j}(t+1)-n_{j}(t) \cong 0(j>0)$. This quasistationary solution is useful when $L_{z}$ is not too large. Within this approximation, the solution of Eq. (4) has the form $n_{j}=A j+B$, so taking into account Eqs. (2) and (3), we have obtained

$$
\begin{gathered}
n_{j}(\theta)=J_{S}(\theta)\left[(j / p)+k_{r}^{-1}(\theta)\right], \\
k_{r}^{-1}(\theta) \equiv\left[\phi^{-1}(\theta)-1\right] / p .
\end{gathered}
$$

Physically, the quantity $k_{r}^{-1}$ defined in Eq. (6) can be interpreted as the resistance due to adsorbed particles [2]. Note that Eq. (5) implies that the flux of particles is related to the concentration in the reservoir $n_{R}$ by $J_{S}(\theta)=K(\theta) n_{R}$, where $K(\theta)$ is the kinetic coefficient given by

$$
K^{-1}(\theta) \equiv\left(L_{z} / p\right)+k_{r}^{-1}(\theta) .
$$

Equation (7) can be interpreted as the combination of a resistance $k_{r}^{-1}(\theta)$ due to the interaction with adsorbed particles in series with an ideal resistance $k_{0}^{-1} \equiv L_{z} / p$ depending only on diffusion (independent of interactions with the substrate). The flux verifies $J_{S} \leqslant J_{0}$, where $J_{0}=k_{0} n_{R}$. We recall that the results given by Eqs. (5) -(7) are the discretized version of the more general results obtained in Ref. [2]. In Fig. 1 we compare the flux obtained in simulations with the qua- 


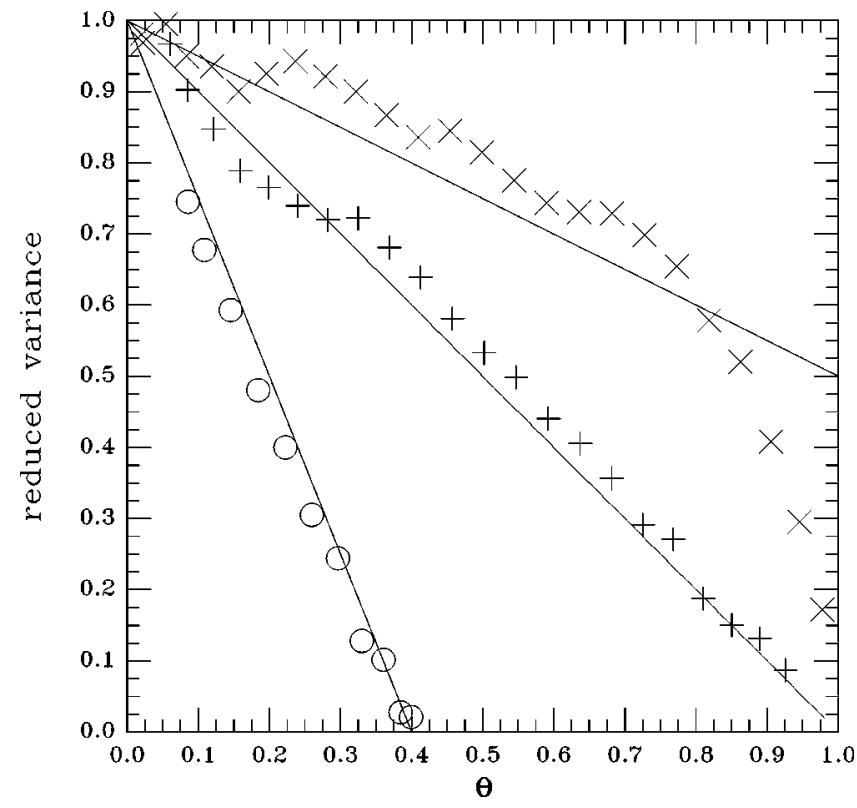

FIG. 2. $V_{r}(\theta)$ in closed cell conditions: simulations $S 1(\times)$, $S 2(+)$, and $S 3(\bigcirc)$; binomial distribution (solid line).

sistationary result given by $J_{S}=K(\theta) n_{R}$. In the case of simulation $S 4$ there is a good agreement between the stationary solution and simulations. In the case of simulation $S 5, L_{z}$ is too large and the quasi-stationary hypothesis fails. In this case, we have solved numerically Eqs. (2)-(4). The nonstationary flux calculated in this way agrees well with simulations.

Fluctuations. The behavior of $V_{r}(\theta)$ (Figs. 2 and 3) shows that fluctuations strongly depend on the boundary and initial conditions imposed on the system. In all the simulations, we have the same transport mechanisms (namely diffusion) and the same interactions between incoming and ad-

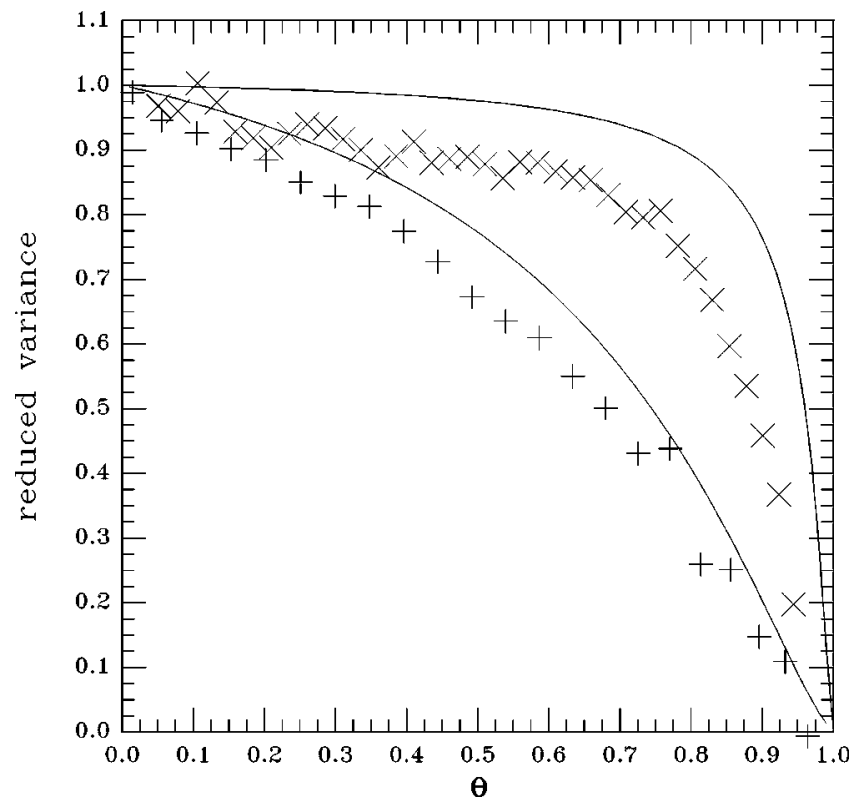

FIG. 3. $V_{r}(\theta)$ in open cell conditions: simulations $S 4(+)$ and $S 5(\times)$ and master equation calculations (solid line). sorbed particles (excluded volume interaction) but the behavior of the reduced variance is very different in each set of boundary conditions.

Closed cell conditions. In this case, fluctuations can be understood by noting that a particle which tries to be adsorbed and is rejected can try again to get adsorbed several times into other sites due to bulk diffusion. Let $q=q(t)$ be the probability that a particle initially in the bulk is not adsorbed ("survives" at bulk) at time $t$. If the surface is near saturation and we have more particles at bulk than the number of free adsorbing sites, it is possible that this particle will not be adsorbed. However, if these conditions are not fulfilled, the particle will adsorb after a short time. In this case, the probability $P\left(N_{0}, t\right)$ that we observe $N_{0}$ adsorbed particles at time $t$ follows the binomial distribution,

$$
P\left(N_{0}, t\right)=\frac{N_{B} !}{N_{0} !\left(N_{B}-N_{0}\right) !}(1-q)^{N_{0}} q^{N_{B}-N_{0}},
$$

where $N_{B}$ is the total number of particles in the system (which is equal to the initial number of particles in the bulk). The mean number of adsorbed particles is $\bar{N}_{0}(t)=(1$ $-q) N_{B}$ and the reduced variance is given by

$$
V_{r}^{b i n}=1-\frac{\bar{N}_{0}}{N_{B}}=1-\frac{N_{\max }}{N_{B}} \theta .
$$

In Fig. 2 we compare Eq. (9) with closed cell simulations. In the case of $S 2$ and $S 3$, simulations agree well with Eq. (9). Thus, excluded volume interactions between incoming and adsorbed particles do not influence $V_{r}$ due to bulk diffusion. However, excluded volume effects are important near the jamming limit. Note that at the jamming limit all the adsorption sites are occupied and no fluctuations are possible $\left(V_{r}\right.$ $=0$ ). But if $N_{B}>N_{\max }$, Eq. (9) vanishes at the unphysical coverage $\theta=N_{B} / N_{\max }>1$. Simulation $S 1$ corresponds to this situation. The simulation results deviates abruptly from Eq. (9) near the jamming limit because $V_{r}$ must vanish at $\theta=1$ (see Fig. 2).

Open cell conditions. In this case, simulations clearly show that fluctuations strongly depend on the distance between the adsorbing surface and the particle reservoir (see Fig. 3). The influence of the reservoir is due to the fact that particles diffusing near the surface can easily return to the reservoir if it is near $j=0$ (as in simulation $S 4$ ). In this case, an incoming particle can explore only a small distance after a failed adsorption attempt. Thus, fluctuations are affected by the combined effect of excluded volume interactions and the reservoir. If the reservoir is far enough from the surface (as in $S 5$ ), $V_{r} \simeq 1$ for not too large $\theta$. Near the jamming limit, $V_{r}$ decreases with $\theta$ due to excluded volume interactions.

In order to obtain approximately $V_{r}(\theta)$, we use the master equation approach developed in Ref. [6]. This approach has been applied successfully to the case of geometrical models. In these models, the diffusion of particles at bulk is not taken into account explicitly but the excluded volume interactions between incoming and adsorbed particles are taken into account in detail. These excluded volume interactions are described by the available surface function $\Phi(\theta)$ that relates 
the adsorption attempts with the flux. Using the master equation approach, $V_{r}(\theta)$ can be computed from $\Phi(\theta)$ [6]. In the CA model in the quasistationary approximation, we can apply the master equation approach if we replace the $\Phi(\theta)$ concept of geometrical models by the kinetic coefficient $K(\theta)$. The kinetic coefficient $K(\theta)$ defined in Eq. (7) generalizes $\Phi(\theta)$ and takes into account the diffusion of incoming particles, the presence of a particle reservoir and the excluded volume interaction. Performing this replacement in Eq. (15) of Ref. [6] we have

$$
V_{r}(\theta)=\frac{K^{2}(\theta)}{\theta} \int_{0}^{\theta} \frac{d \theta^{\prime}}{K^{2}\left(\theta^{\prime}\right)}
$$

We have obtained an explicit analytic expression for $V_{r}(\theta)$ by substituting Eq. (7) in Eq. (10) and performing the integration. The obtained result verifies the expected limiting behaviors: $V_{r}(\theta=1)=0$ and $\lim _{z_{R} \rightarrow \infty} V_{r}(\theta)=1$. For small $\theta$, we have obtained, from Eqs. (7) and (10), $V_{r}=1-\theta / L_{z}$ $-\theta^{2}(4 / 3)\left(L_{z}-1\right) / L_{z}^{2}+O\left(\theta^{3}\right)$. In Fig. 3 we have evaluated Eqs. (7) and (10) for the parameters corresponding to simulations $S 4$ and $S 5$. Qualitatively, the behavior of $V_{r}(\theta)$ is well reproduced by Eq. (10). However, Eq. (10) overestimates $V_{r}$ for large $L_{z}$ due to the failure of the stationary hypothesis on which Eqs. (7) and (10) are based.

In summary, we have observed that the roles played by bulk diffusion and excluded volume effects on coverage fluctuations depend strongly on the boundary conditions.

The results of the CA model in the case of closed cell boundary conditions show that the fluctuations in the number of adsorbed particles can be described by a simple binomial distribution except near the jamming limit. This is a consequence of the bulk diffusion of incoming particles. Excluded volume effects influence $V_{r}$ only near the jamming limit if the initial number of particles in the system is much larger than the number of sites available for adsorption. In the case of open cell boundary conditions, fluctuations strongly depend on the position of the particle reservoir (which determines the distance which a particle can travel by diffusion). If the particle reservoir is maintained far enough from the adsorbing surface, bulk diffusion dominates and the reduced variance is nearly 1 even for relatively high coverages. If the particle reservoir is near the adsorbing surface, $V_{r}$ is strongly influenced by excluded volume interactions. In this case, we have obtained an analytical expression for $V_{r}(\theta)$ by extending to the CA model of the master equation theory presented in Ref. [6].

In recent experimental studies [3-5], the behavior of $V_{r}(\theta)$ has been analyzed as a function of the transport mechanisms and interactions between incoming and adsorbed particles. However, our model clearly shows that the role played by these factors on fluctuations strongly depends on the boundary conditions imposed. Thus, our results have to be taken into account in order to understand properly the effect of these factors on the experimental results.
[1] P. Schaaf et al., Ann. Phys. (Paris) 23, 1 (1998); Z. Adamczyk et al., Adv. Colloid Interface Sci. 48, 151 (1994).

[2] J. Faraudo and J. Bafaluy, Europhys. Lett. 46, 505 (1999); J. Chem. Phys., 112, 2003 (2000).

[3] P. Lavalle et al., Proc. Natl. Acad. Sci. U.S.A. 96, 11101 (1999).
[4] P. Wojtaszczyk et al., J. Chem. Phys. 103, 8285 (1995).

[5] Z. Adamczyk et al., J. Chem. Phys. 105, 5552 (1996); 113, 11 336 (2000).

[6] J. Bafaluy et al., J. Chem. Phys. 107, 2089 (1997).

[7] B. Chopard and M. Droz, J. Stat. Phys. 64, 859 (1991). 